\title{
Datenerfassung und Visualisierung von Vitalparametern und Primärsignalen auf dem Computer und auf mobilen Endgeräten
}

\author{
Ralf Vandenhouten, Thomas Behrens, Miriam Selz
}

\begin{abstract}
Zusammenfassung
In Kliniken besteht der Bedarf nach einer lückenlosen Aufzeichnung der Vitalparameter (z. B. Herzfrequenz, Blutdruck) und Primärsignale (z. B. EKG). Besonders in der Notfallmedizin ist eine ständige Patientenüberwachung lebensnotwendig. Zudem dienen die erfassten Daten der aktuellen Patienten-Dokumentation, aber auch zur Analyse im Bereich der Forschung und für Studien. Für diesen Zweck wurde das Telematik-System »Multi-Patientenmonitoring « entwickelt. Dieses System dient der Datenerfassung, Signalvisualisierung und Echtzeitübertragung von Vitalparametern an beliebig viele PCs und mobile Geräte des Krankenhausnetzwerkes.

Motiviert durch das Universitätsklinikum Tübingen wurde das Projekt von der Wildauer Firma ixellence initiiert und in interdisziplinärer Zusammenarbeit mit medizinischen Einrichtungen, dem Fachgebiet Telematik an der TFH Wildau und der Philips Medizin-Systeme $\mathrm{GmbH}$ realisiert.
\end{abstract}

\begin{abstract}
Hospitals need a complete recording of vital parameters (e.g. heart frequency, blood pressure) and primary signals (e.g. ECG). Especially in intensive care units the permanent monitoring and the recording of the current status of the patient is vital. Furthermore, these data are useful for the patient documentation and in fields of research and studies. For this purpose a multi-monitoring system was developed. This system provides the data acquisition, signal visualization and real time transmission of vital parameters to PCs and mobile devices in the hospital network. With this system it is possible to get a fast overview of the current patient status and its history. Furthermore physicians can monitor the patients mobile and in real time.

The project was developed in co-operation with medical research facilities, the Chair of Telematics at the University of Applied Sciences in Wildau, and with the companies Philips Medical Systems and ixellence.
\end{abstract}

\section{1 Überblick und Funktionsweise}

In Kliniken besteht der Bedarf nach einer lückenlosen Aufzeichnung der Vitalparameter (z. B. Herzfrequenz, Blutdruck) und Primärsignale (z. B. EKG). In Verbindung mit einer entsprechend visualisierten Darstellung unter Einbeziehung der Historie soll diese einen schnellen Überblick zur Beurteilung des aktuellen Patientenstatus liefern. Nach einer Aufzeichnung der Daten über einen längeren Zeitraum sollen außerdem retrospektiv einzelne Ereignisse genauer analysiert oder langzeitarchiviert werden können.

Das hier vorgestellte plattformunabhängige Telematik-System für das Patientenmonitoring ist darauf ausgerichtet, diese Anforderungen zu erfüllen. Es liest Daten von Überwachungsmonitoren aus, wertet diese aus, visualisiert und archiviert sie. Insbesondere ist es mit dem ergänzenden Monitoring-System nun möglich, hochauflösende Vitalparametertrends darzustellen und zurückliegende Abschnitte von Primärsignalen zu inspizieren. Die ausgelesenen Daten werden dem Anwender ortsunabhängig und zu jedem Zeitpunkt über das lokale Netzwerk sowie im Falle mobiler Einheiten über WLAN zur Verfügung gestellt. Mit Hilfe einer entsprechend visualisierten Darstellung wird ein schneller Überblick über den aktuellen Status des Patienten, über Trends oder historische Werte geliefert.

Bei der Datenvisualisierung kann, neben einer Trenddarstellung der Vitalparameter, zu jedem beliebigen Zeitpunkt mittels einer Zoomfunktion auf die Primärsignale zurückgegriffen werden. Für die historische Sicht werden die anzuzeigenden Daten aus der zentralen Datenbank geladen. Damit die Primärsignale kontinuierlich aktualisiert angezeigt werden können (Echtzeitübertragung), werden die aktuellen Daten nicht zeitverzögert aus der zentralen Datenbank geladen, sondern direkt vom zentralen Datenerfassungsmodul geholt. Die Applikation für mobile Endgeräte (wie PDAs) ermöglicht zudem die Ausgabe akustischer Alarme.

Der Arzt ist mit diesem System in der Lage, seine Patienten umfassend zu überwachen. Er kann von jedem Ort im Krankenhaus den Zustand des Patienten einsehen. Besonders auf Intensivstationen und im Bereich der Altenpflege kommt das mobile Patientenmonitoring-System sinnvoll zum Einsatz. Bei der Betreuung der extrem pflegeintensiven Patienten ist ein agiler und flexibler Per- 
sonaleinsatz unerlässlich. Die mobile Überwachung mit dem PDA kann diese Arbeit erheblich unterstützen. Des Weiteren lassen sich durch Aufzeichnung der Daten über einen längeren Zeitraum nachträglich einzelne Ereignisse genauer analysieren. Diese Daten werden zum einen für die aktuelle Patienten-Dokumentation, zum anderen aber auch vermehrt im Bereich der Forschung genutzt.

\section{Aufgabe}

Die Aufgabe bestand darin, Patientendaten hochauflösend und in Echtzeit direkt auf dem PC zu visualisieren und zu speichern. Um ein Multi-Patientenmonitoring und die Fernvisualisierung zu ermöglichen, sollte das lokale Netzwerk (LAN und WLAN) genutzt werden. Zudem sollte aber auch eine direkte Verbindung zu den Überwachungsgeräten aufgebaut werden können [12].

Mit der Entwicklung einer mobilen Komponente sollte der aktuelle Status eines Patienten auch auf einem PDA beobachtet werden können.

Speziell im intensivmedizinischen Bereich, zur Führung einer Narkose und zur Überwachung von Intensivpatienten, ist es neben der Anzeige der aktuell gemessenen Primärsignale und Vitalparameter notwendig, auch Verläufe von Vitalparametern, sog. Trends, zu beurteilen [13, 14]. Moderne Überwachungsmonitore erlauben die Kontrolle der wichtigsten Vitalparameter in unmittelbarer Nähe des Patientenbetts, sind aber in ihren Darstellungsmöglichkeiten begrenzt. Zudem stellen sie reine Überwachungsgeräte dar und speichern Patientendaten nur über maximal 48 Stunden und in geringer Abtastrate [1]. Besteht eine Vernetzung der Monitore, inklusive einer zentralen Überwachungsanlage, sind Aufzeichnungen bis zu 96 Stunden möglich. Zur Erfüllung der Beweispflicht bei unvorhergesehenen Ereignissen ist aber auch dies $\mathrm{u}$. U. nicht ausreichend. Hier ist es erforderlich, hochauflösende Daten über längere Zeiträume zur Weiterverarbeitung abspeichern zu können und in Einzelfällen die in diesem Zusammenhang angefallenen Informationen zu analysieren oder auszudrucken.

Es besteht die Möglichkeit, Daten aus der Überwachungszentrale über eine HL7-Schnittstelle (Health Level 7) auszulesen. HL7 ist ein internationaler Standard für den Datenaustausch im Gesundheitswesen [2], unterstützt aber nur die Übertragung von Signalen mit niedriger zeitlicher Auflösung und überträgt diese nur alle zehn Sekunden. Eine Archivierung hochauflösender Daten und ein Review sind somit nicht möglich $[3,4]$.

Zusammengefasst waren folgende Anforderungen zu berücksichtigen:

- Erfassung, Speicherung und Visualisierung von Vitalparametern und Primärsignalen

- Plattformunabhängigkeit

- Client/Server-Architektur

- Zentrale/dezentrale Datenerfassung

- Zentrale Speicherung der erfassten Daten

- Unabhängige Client-Anwendung zur Visualisierung und Auswertung der Daten

- Datenschutz: Zugriffsverwaltung benutzerorientiert

\section{Architektur und System}

Für die Vernetzung der Komponenten wurde ein WWWund WAP-basiertes Telematik-System zur Unterstützung der Fernüberwachung und -steuerung von vernetzten Geräten zugrunde gelegt, das im Rahmen eines Forschungsprojektes im Fachbereich Telematik der TFH Wildau entwickelt worden war [8].

Um sowohl eine Einzelplatzlösung als auch eine Mehrplatzlösung mit verteilter Last zu erreichen, war es wichtig, dass die drei Ebenen der Datenverarbeitung (Datenerfassung, -speicherung und -visualisierung) strikt voneinander getrennt sind.

Hierdurch lässt sich flexibel sowohl ein Einzelplatzsystem, bei dem alle drei Komponenten auf einem Rechner laufen, als auch ein verteiltes Mehrplatzsystem (Abb. 1) aufbauen, bei dem neben mehreren Client-Instanzen des Anwenderprogramms auch eine flexible Anzahl von Patientenmonitoren eingebunden werden kann. Die einzelnen Komponenten müssen sich kooperativ mit anderen Anwendungen auf dem jeweiligen Rechner die lokalen Ressourcen teilen. Dies gilt auch für die Netzwerkbelastung.

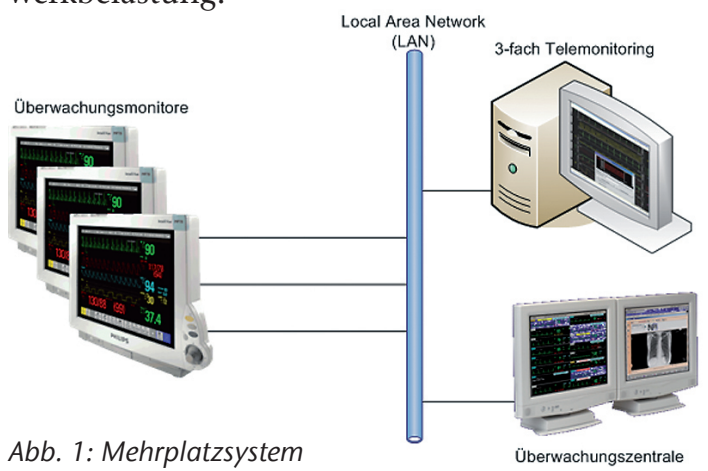

Zunächst wurde eine Software-Architektur für das System konzipiert, die aus einem Schichtenmodell mit fünf Schichten besteht (Abb. 2). [6, 10] Ziel dabei war, flexibel unterschiedlichste Endgeräte einbinden zu können. $\mathrm{Zu}$ den Geräten gehören solche, die eine Verbindung zu einem Computer haben und deren Daten ausgewertet und/oder aus der Ferne visualisiert werden sollen, im vorliegenden Fall Patientenmonitore.

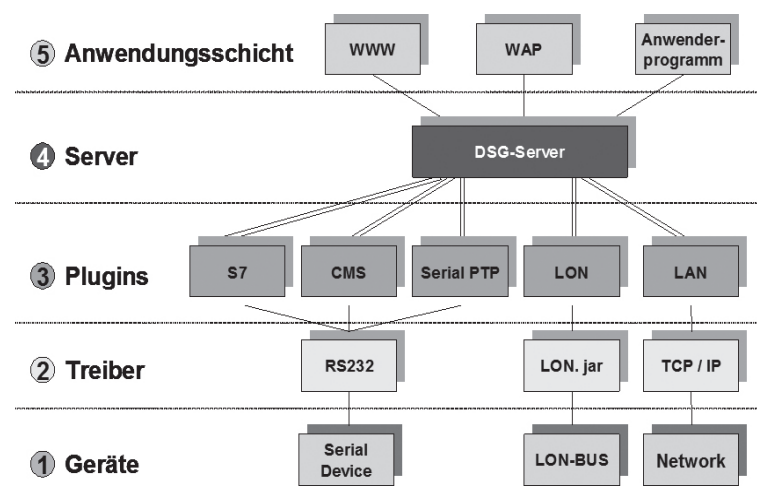

Abb. 2: Schichtenmodell des Systems

Die Treiber sind für die Anbindung der Geräte an die Software über eine Hardwareschnittstelle verantwortlich, zum Beispiel über RS232 (serielle Schnittstelle) oder TCP/IP. 
Um Daten aus den Geräten zu lesen, werden (in der Regel proprietäre) Protokolle verwendet. Diese Protokolle sind für verschiedene Geräte unterschiedlich und sowohl vom Hersteller als auch von der Art und der Menge der gelieferten Daten abhängig. Die Plug-Ins kommunizieren mit den Geräten über deren Protokolle und konvertieren die Daten in allgemeine Datenstrukturen, die der Server verarbeiten kann. Für die unterschiedlichen Geräte werden demzufolge verschiedene Plug-Ins benötigt. Die Plug-In-Schicht macht die spezifischen Eigenheiten einzelner Geräte für den Server transparent.

Der Server ist das Kernstück des Systems. Dieser verwaltet die Daten der Geräte und stellt die Benutzerschnittstellen bereit, über die die Daten geschrieben und gelesen werden können.

In der Anwendungsschicht werden die Daten visualisiert. Das kann das Darstellen von Messdaten, Zeitreihen oder mehrdimensionalen Messdaten sein.

\section{Datenerfassung und Speicherung}

Die unabhängige Datenerfassung unterstützt folgende Szenarien:

1. zentrale Datenerfassung von einem oder mehreren Überwachungsgeräten mit lokaler Datenspeicherung (Abb. 4)

2. dezentrale Datenerfassung von einem bzw. mehreren Überwachungsgeräten mit zentraler Datenspeicherung. (Abb. 3)

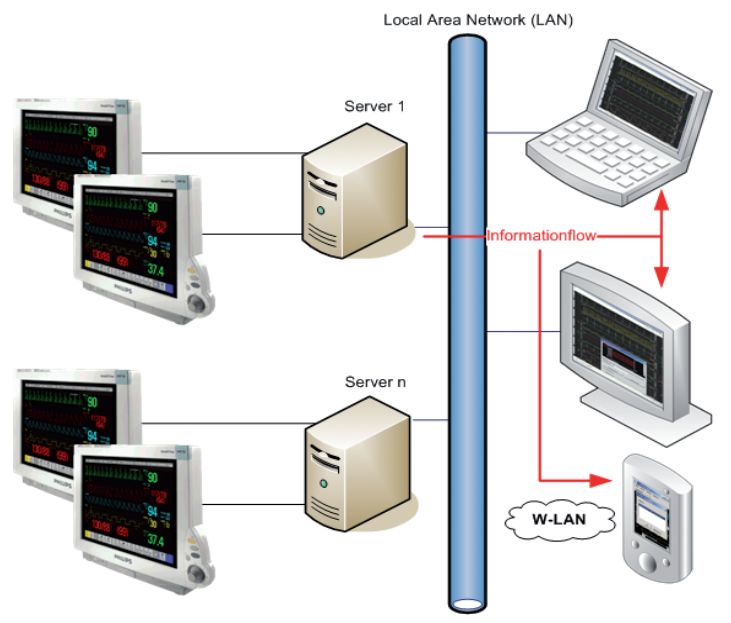

Abb. 3: Netzwerklösung mit lokaler Speicherung

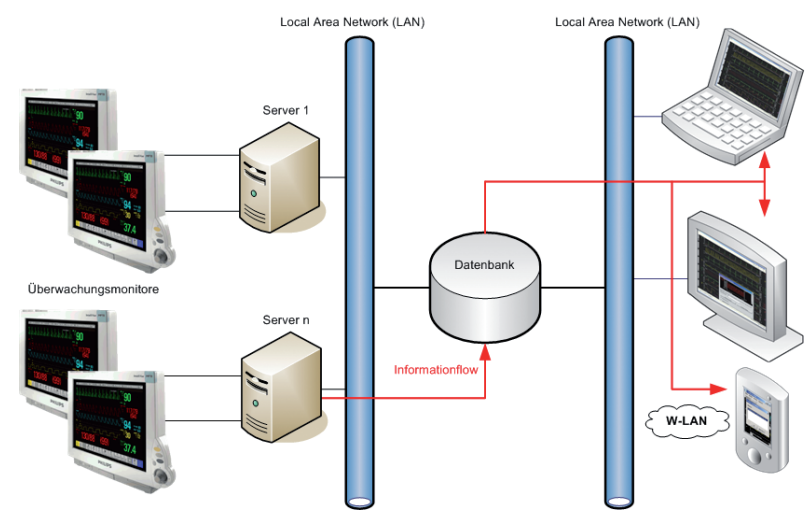

Abb. 4: Zentrale Datenbanklösung
Die Daten stellt der Überwachungsmonitor z. B. über seine serielle Schnittstelle (RS232) zur Verfügung. Die Daten sind vom Typ »numeric « (Daten mit niedriger zeitlicher Auflösung, die am Patientenmonitor numerisch angezeigt werden) oder »wave « (Daten mit hoher Abtastrate, die als Kurven visualisiert werden), die Übertragungsrate beträgt 115200 bps. Da die Abtastintervalle der hochaufgelösten wave-Signale im Millisekundenbereich liegen, ist die Anzahl der synchron erfassbaren Signale vom Monitor bzw. dessen Schnittstelle her begrenzt. So können maximal 12 wave-Signale (z. B. EKG oder Blutdruck), und alle numerischen Signale (wie z. B. Puls oder $\mathrm{CO}_{2}$-Konzentration im Blut) zur selben Zeit erfasst werden.

Die Signale haben die folgenden Abtastraten:

- ein normales EKG-Signal (wave): 2 ms

- ein zusammengesetztes EKG-Signal (wave): $4 \mathrm{~ms}$

- ein nicht-EKG wave-Signal, wie Blutdruck: 8 ms oder $16 \mathrm{~ms}$

- ein numerisches Signal (numeric): $1 \mathrm{~s}$

Beim Speichern der Signale ergeben sich daraus die folgenden Datenmengen nach einer Stunde:

- ein normales EKG-Signal: ca. 7,5 MB

- ein zusammengesetztes EKG-Signal: ca. 4 MB

- ein nicht-EKG Wave-Signal: ca. 2,25 MB (8 ms), ca. $1,35 \mathrm{MB}$ (16 ms)

- ein Numeric-Signal: ca. $125 \mathrm{~KB}$

Die Erfassung und Archivierung der Signale mit hoher Abtastrate (wave) in ihrer höchstmöglichen Auflösung sind für die Anwender von großer Bedeutung, insbesondere für Analysezwecke und für die Beweispflicht. Für die Entwicklung des Systems stellten diese Datenmengen für die Performance (Übertragungsgeschwindigkeit, Datenverarbeitung und -speicherung) eine große Herausforderung dar, insbesondere im Hinblick auf das Datenbanksystem (Kapitel 5.2.1).

\section{Anwenderprogramm}

\subsection{Datenvisualisierung}

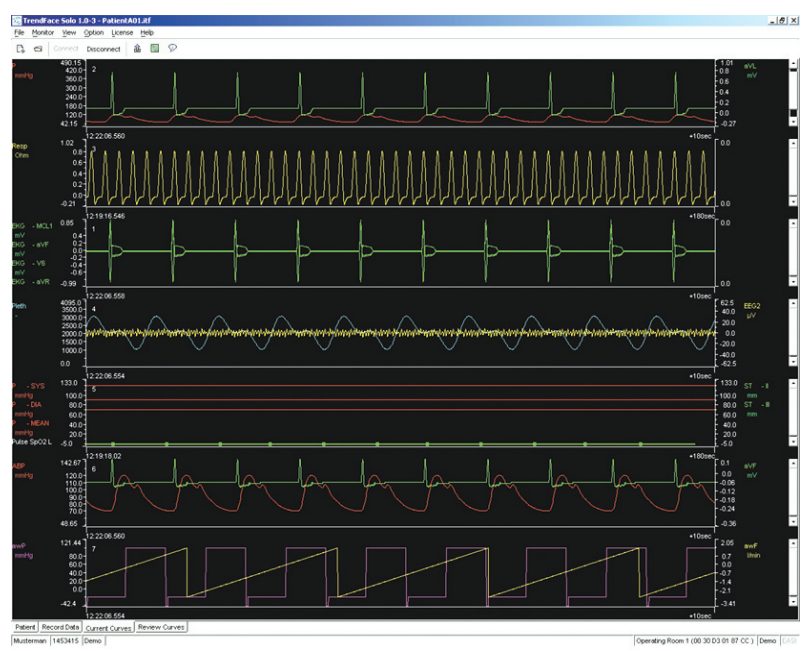

Abb. 5: Screenshot des Anwenderprogramms auf dem PC, Visualisierung der Echtzeitdaten 
Bei der Datenvisualisierung kann neben einer Trenddarstellung der Vitalparameter (Abb. 5) zu jedem beliebigen Zeitpunkt auch auf die Primärsignale zurückgegriffen werden. Die anzuzeigenden Daten, aktuelle oder historische, können aus der zentralen Datenbank oder direkt vom lokalen Datenerfassungsmodul geladen werden. Das Anwenderprogramm zeigt beim Start automatisch die Daten für einen bestimmten Patienten bzw. ein Bett an. Da die Patientenmonitore »Citrix-fähig « sind, d. h. entfernte Applikations- und Terminalserver-Anwendungen starten und nutzen können, bestand zudem die Forderung, das Telemonitoring-Programm an diese Funktionalität anzupassen. Deshalb ist ein flexibler Aufbau des vernetzten Systems realisiert worden, bei dem die webfähige Anwendung auch direkt vom Patientenmonitor gestartet werden kann. Die Daten können von hier aus erfasst und in der zentralen Datenbank (Abb. 4) archiviert werden.

\subsection{Funktionen}

Die Art der Signaldarstellung ist vielfältig konfigurierbar. Beim nächsten Programmstart werden die letzten Einstellungen automatisch wiederhergestellt. Für einen Wechsel zwischen verschiedenen Betriebsmodi können komplette Einstellungsprofile gespeichert werden. Wenn die Erfassung an das lokale Netzwerk angeschlossen wird, gibt die Anwendung die Möglichkeit, Patientendaten gleichzeitig an mehreren Rechnern auszuwerten. Man sieht an einem Rechner alle an das lokale Netzwerk angeschlossenen Patientenmonitore.

\subsubsection{Funktionsumfang der Einzelplatzlösung}

Die wichtigsten Funktionen der Einzelplatzlösung sind:

- Aufzeichnen der Vitalparameter und Primärsignale

- Darstellung des aktuellen Status des Patienten

- periodische Abfrage der zu liefernden Daten

- retrospektive Sicht auf lokal archivierte Daten

- Exportieren der Daten in das universelle CSV-Format

- Trend-Aufzeichnung über einen längeren Zeitraum und Analyse mit Datenanalysetools

\subsubsection{Funktionsumfang der Netzwerklösung} Zur Einzelplatzlösung kommen bei der Netzwerklösung folgende Funktionen hinzu:

- Sicht auf die zurückliegenden Patientendaten

- Multi-Monitoring - ein Patient/Bett zu mehreren PCs oder Monitoren (Citrix-fähige Anwendung)

- Verbindung mehrerer PCs über das lokale Netzwerk

- Patientendaten können gleichzeitig an mehreren Rechnern ausgewertet werden

- Sicht auf die aktuellen Daten auch auf dem PDA (Abb. 6)

- einfache Migration zur Datenbankversion

\subsubsection{Funktionsumfang der Datenbanklösung} Um die Daten zu archivieren, wurden zwei Möglichkeiten geschaffen, zum einen eine dateibasierte Datenbank und zum anderen eine relationale SQL-Datenbank. Beide Möglichkeiten haben Vor- und Nachteile. Die dateibasierte Datenhaltung ist genau auf die Anforderungen und
Daten der Telemonitoring-Applikation zugeschnitten. So können die Daten effektiv und performant geschrieben und gelesen werden. Zudem ist der Speicherplatzbedarf darauf optimiert. Die Werte der Kurven werden nicht jeweils mit Zeitstempel gespeichert, sondern blockweise, so dass nur ein Zeitstempel und die Zeitabstände zwischen den einzelnen Werten in einem Block vorhanden sind.

Da dieses System aber propritär und somit schlecht oder umständlich von externen Anwendungen auszuwerten ist, können die Daten alternativ auch in einer relationalen SQL-Datenbank gespeichert werden. Die relationalen SQL-Datenbanken können nicht 100-prozentig auf das Telemonitoring-System zugeschnitten werden, so sind gegenüber der dateibasierten Datenbank ein höherer Speicherplatzbedarf und Prozessorleistungsbedarf erforderlich. Die Daten können aber von externen Systemen mit dem veröffentlichten ER-Modell gelesen und ausgewertet werden. Weiterhin bieten SQL-Datenbanken Transaktionssicherheit und Backupmechanismen, die eine größere Robustheit des Systems gewährleisten.

Um die Performance der SQL-Datenbank zu verbessern, wurden während der Entwicklung einige Perfomancetests mit unterschiedlichen ER-Modellen und SQL-Datenbanken durchgeführt. Dabei hat sich herausgestellt, dass die Werte der Wave-Kurven auch, wie bei der dateibasierten Datenbank, blockweise gespeichert werden müssen. Dafür werden in der Datenbank BLOBDatentypen verwendet. Die einzelnen Werte werden binär und sequentiell in einem Datensatz (Zeitstempel, Anzahl Werte, Zeitintervall zwischen den Werten) gespeichert. Gegenüber der Verwendung eines »normalen « Datentyps, wie z. B. »float«, sind die Performance ca. zehnmal langsamer und der Speicherplatzbedarf um einiges höher, da zu jedem Wert der Zeitstempel und mindestens die Referenz zu dem Patienten gespeichert bzw. gelesen werden muss.

\section{PDA-Software}

Um Signale auch auf mobilen Geräten bereitstellen zu können, wurde eine prototypische Java-Applikation für PDAs mit entsprechender drahtloser Anbindung an das System entwickelt (Abb. 6). Im Gegensatz zum stationären Telemonitoring, bei dem die Langzeitarchivierung eine wichtige Rolle spielt, stand bei der mobilen Anwendung die aktuelle Überwachung der Patienten im Vordergrund, die besonders im intensivmedizinischen Bereich von Bedeutung ist.

Das mobile System ist über das interne lokale Funknetz (WLAN, Standard IEEE 802.11b) mit dem Server verbunden. Mit Hilfe des Servers werden die Verbindung zur hausinternen Infrastruktur (über LAN) hergestellt und die erfassten Daten übertragen, um auf dem PDA visualisiert werden zu können. Der Zugriff vom PDA auf die Daten des Servers erfolgt über RMI (Remote Method Invocation).

Von großer Bedeutung für die Anwender war eine handhabbare und benutzerorientierte Anwendung und Darstellung auf dem PDA. Deshalb wurden in enger $\mathrm{Zu}-$ 


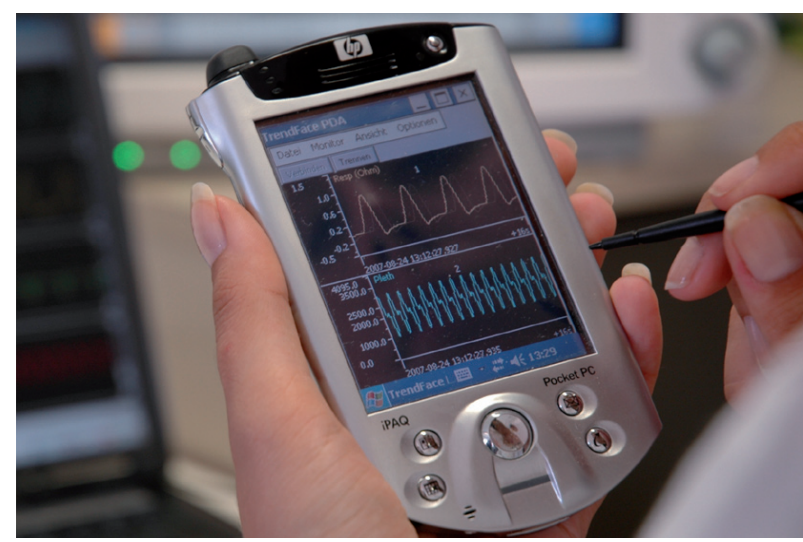

Abb. 6: Bild der mobilen Lösung

sammenarbeit mit dem Universitätsklinikum Tübingen nur ausgewählte Funktionen in die mobile Anwendung übernommen. Dem mobilen Anwender stehen die Daten und Signale der an den Monitoren angeschlossenen Patienten über WLAN zur Verfügung. Die Patienten werden anhand des Monitornamens ausgewählt (li. Screenshot Abb. 7). Anschließend können die gewünschten Signale aus einer Liste ausgewählt werden. Der dritte Screenshot der Abb. 7 zeigt die Visualisierung der ausgewählten Signale.
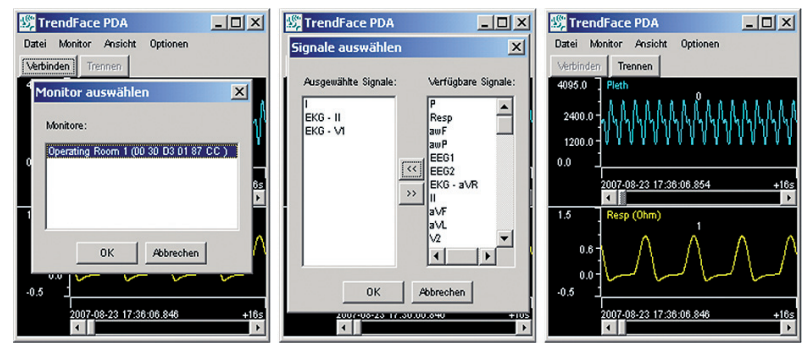

Abb. 7: Screenshorts der mobilen Lösung

Eine Besonderheit der mobilen Anwendung ist die akustische Ereignismeldung. Das Programm holt sich auch die Alarme der Monitore (= wichtige Ereignisse) vom Server. Entsprechend akustisch hinterlegt, werden diese Ereignisse und ihre mögliche Ursache angezeigt. Tritt ein Monitor-Ereignis ein, werden die ursächlichen Signale in der Datenbank bis zur Bestätigung des Alarms direkt am Patientenmonitor gespeichert. Dies hilft dem Arzt, Ereignisse im Review genauer zu analysieren.

\section{Kooperation, Technologietransfer und Weiterentwicklung}

Der Weg zur Softwarelösung war durch eine enge Abstimmung zwischen der TFH Wildau und den Partnern ixellence, Philips Medizin-Systeme und der Uniklinik Tübingen geprägt. Nur so konnte sichergestellt werden, dass ein System entwickelt wurde, das den Anforderungen der Kliniken gerecht wurde und sich in der Praxis bewährt.

Der Studiengang Telematik der TFH Wildau und ixellence kooperieren schon seit einiger Zeit in verschiedenen Forschungsprojekten. Aus der erfolgreichen $\mathrm{Zu}$ sammenarbeit entwickelte sich auch dieses nationale
Drittmittelprojekt. Hierbei entwickelte das Fachgebiet Telematik das mobile System und unterstützte ixellence bei der Entwicklung einer geeigneten Architektur und der Datenbank.

Der Studiengang Telematik beschäftigt sich mit der Entwicklung von Telematiksystemen, darunter mobile Systeme und Anwendungen für den Businessbereich, auch mit handelsüblichen Funktelefonen. Zudem besteht Expertise mit verschiedenen Verfahren in der Bildverarbeitung und Datenanalyse [5] [9]. In diesem Bereich hat sich eine erfolgreiche Zusammenarbeit zwischen regionalen und nationalen Unternehmen und dem Fachgebiet Telematik der TFH Wildau etabliert.

Die TFH Wildau ist seit 2004 als Aussteller innovativer Systeme auf dem Technologie-Tag in Teltow vertreten. Dort wurde das Patientenmonitoring-System im März 2007 vorgestellt.

ixellence ist schon seit einigen Jahren offizieller Praxispartner des Telematik-Studienganges und bietet Studierenden die Möglichkeit, theoretisch Erlerntes praxisnah umzusetzen. Während der Praxisabschnitte konnten vier Studenten mit Entwicklungsaufgaben aus diesem Projekt betraut werden.

Die Durchführung dieses Projektes und die daraus entstandene enge Beziehung zwischen den beteiligten Partnern sind ein Beispiel für die erfolgreiche Kooperation zwischen Wissenschaft und Wirtschaft. 2005 begann das Gesamtprojekt und wird von ixellence in Zusammenarbeit mit dem Studiengang Telematik stetig weiterentwickelt. So wird gegenwärtig die Weiterentwicklung durch eine Masterarbeit unterstützt. Darin wird untersucht, welche neuen Software-Technologien das TelemonitoringSystem noch flexibler und skalierbarer machen können. Neue Anforderungen, wie die Vernetzung von bis zu 200 Monitoren oder die Anbindung von Monitoren unterschiedlichen Typs, waren Anlass für diese Überlegung.

\section{Zusammenfassung}

Bei der Entwicklung der Applikationen wurde konsequent auf moderne und leistungsfähige Technologien gesetzt. Das Monitoring-System ist modular und wurde in der plattformunabhängigen Sprache »Java « entwickelt.

Es wurde ein Telematik-System entwickelt, das die Fernüberwachung und -steuerung von vernetzten Geräten unterstützt und die Sicht auf aktuelle sowie historische Patientendaten zulässt, ob vom PC, PDA oder vom Monitor aus, ob über LAN, WLAN oder Internet.

Innerhalb eines Netzwerkes richten lokale PCs Anfragen an den Server. Der Server sendet die Daten über das TCP/IP-Protokoll zum Client. Das System bietet ein Multi-Monitoring über PC, Laptop oder PDA, mit dem der Arzt alle an das lokale Netzwerk angeschlossenen Patientenmonitore sehen und überwachen kann. Zudem können die Patientendaten gleichzeitig an mehreren Rechnern ausgewertet werden. Das medizinische Personal hat damit von jedem Ort im Krankenhaus, oder sogar von außerhalb, mobilen oder stationären Zugriff auf alle Vitaldaten. 


\section{Referenzen}

[1] Agilent Technologies Deutschland GmbH, Users Guide Agilent Monitors, Agilent Measurement Server and Agilent Measurement Server Extensions, 2000

[2] Approved American National Standard (ANSI), Health Level Seven Inc., HL7 Messaing Standard Version 2.5, 2003

[3] Koninklijke Philips Electronics N.V., »IntelliVue« Patient Monitor, Data Export Interface Programming Guide, 2003

[4] Philips Medical Systems, HL7 Parameter Data Interface Programmer's Guide, Standard HL7 Version 2.3.1

[5] Vandenhouten, R., Analyse instationärer Zeitreihen komplexer Systeme und Anwendungen in der Physiologie (Analysis of instationary time series of complex systems and applications in physiology), Shaker-Verlag 1998

[6] Vandenhouten, R., Qualitäts- und Ressourcenmanagementsystem für die Produktion auf der Basis von WWW und WAP, Innovationskatalog 2003, BMWA 2003

[7] Vandenhouten, R./Selz, M., Identification and tracking of goods with the mobile phone, IEEE International Conference: International Symposium on Logistics and Industrial Informatics (LINDI 2007), 2007

[8] Vandenhouten, R./Behrens, T., Ein Gatewaysystem für telematikbasiertes Gerätemonitoring, Wissenschaftliche Beiträge der Technischen Fachhochschule Wildau 2004

[9] Vandenhouten, R./Behrens, T./Selz, M., Multi-Telemonitoring: Datenerfassung und Aufzeichnung von Vitalparametern und Primärsignalen auf dem PC oder PDA, 2006, Themenspecial Telematik im Gesundheitswesen, Competence Site/BITKOM, 2006

[10] Behrens, T., Entwicklung eines Gatewaysystems für WWW- und WAP-basiertes Gerätemonitoring, Diplomarbeit TFH Wildau/Universität Potsdam, 2003

[11] Sun Microsystems, The Java API: RMI, http://java.sun. com/j2se/1.4/docs/api

[12] Bleicher, W., Lastenheft Projekt Trendface, Version 1.3. Universitätsklinikum Tübingen, Medizinische Informationstechnologie, 2006

[13] Bleicher, W., Epple, Überlegungen zur Alarmgebung in der Intensivmedizin und in der Narkoseüberwachung, Anästh. Intensivther. Notfallmed. 21 132-136, 1986

[14] Bleicher, W. et al, Artifact Processing in a Computerized Intensive Care Unit, Comp. Crit. Care Pulm. Med., Springer. 107-115, 1985

\section{Literatur}

Agilent Technologies Deutschland GmbH, Users Guide Agilent Monitors, Agilent Measurement Server and Agilent Measurement Server Extensions, 2000

Approved American National Standard (ANSI), Health Level Seven Inc., HL7 Messaing Standard Version 2.5, 2003

Behrens, T., Entwicklung eines Gatewaysystems für WWWund WAP-basiertes Gerätemonitoring, Diplomarbeit TFH Wildau/Universität Potsdam, 2003
Bleicher, W. et al. Artifact Processing in a Computerized Intensive Care Unit. Comp. Crit. Care Pulm. Med., Springer. 107-115, 1985

Bleicher, W., Epple, Überlegungen zur Alarmgebung in der Intensivmedizin und in der Narkoseüberwachung, Anästh. Intensivther. Notfallmed. 21 132-136, 1986

Bleicher, W., Lastenheft Projekt Trendface, Version 1.3. Universitätsklinikum Tübingen, Medizinische Informationstechnologie, 2006

Koninklijke Philips Electronics N.V., »IntelliVue« Patient Monitor, Data Export Interface Programming Guide, 2003

Philips Medical Systems, HL7 Parameter Data Interface Programmer's Guide, Standard HL7 Version 2.3.1

Sun Microsystems, The Java API: RMI, http://java.sun.com/ j2se/1.4/docs/api

Vandenhouten, R./Behrens, T./Selz, M., Multi-Telemonitoring: Datenerfassung und Aufzeichnung von Vitalparametern und Primärsignalen auf dem PC oder PDA, 2006, Themenspecial Telematik im Gesundheitswesen, Competence Site/BITKOM, 2006

Vandenhouten, R./Behrens, T., Ein Gatewaysystem für telematikbasiertes Gerätemonitoring, Wissenschaftl. Beiträge TFH Wildau, 2004

Vandenhouten, R., Qualitäts- und Ressourcenmanagementsystem für die Produktion auf der Basis von WWW und WAP, Innovationskatalog 2003, BMWA, 2003

Vandenhouten, R./Selz, M., Identification and tracking of goods with the mobile phone, IEEE International Conference: International Symposium on Logistics and Industrial Informatics (LINDI 2007), 2007

Vandenhouten, R., Analyse instationärer Zeitreihen komplexer Systeme und Anwendungen in der Physiologie (Analysis of instationary time series of complex systems and applications in physiology), Shaker-Verlag 1998

\section{Autoren}

Prof. Dr. rer. nat. Ralf Vandenhouten

Technische Fachhochschule Wildau

Fachgebiet Telematik

Tel. +493375 508-359

ralf.vandenhouten@tfh-wildau.de

\section{Dipl.-Inform. Thomas Behrens}

Miriam Selz, B. Eng.

Wissenschaftliche Mitarbeiterin

Technische Fachhochschule Wildau

Fachgebiet Telematik

Tel. +493375 508-616

miriam.selz@tfh-wildau.de 